\title{
De la littérature comme économie du plaisir.
}

\section{Cristina Robalo-Cordeiro*}

FLUC

Résumé : Envisagée d’abord sous l'aspect de l'économie politique, la littérature se présente comme un secteur de l'industrie du livre, les termes de production et de consommation pouvant désigner avec exactitude les rapports entre les auteurs (assistés des éditeurs, imprimeurs, libraires...) et les lecteurs, le plaisir de ces derniers étant tenu pour la cause finale de ce commerce. Mais si nous restreignons la perspective au livre seul, il est encore légitime, considérant par exemple "l'économie » d'un récit ou d'un poème, d'y découvrir une gestion du plaisir de lire, allant de l'incitation à l'excitation maximale, comme dans une psychanalyse du feu. Reste à se demander si le modèle sexuel peut rendre fidèlement compte du type de satisfaction que procure la lecture. N'est-ce pas plutôt du côté de la sublimation qu'il faut chercher le secret de la lecture telle que l'encourage la Société dans sa préconisation des activités reconnues d'utilité publique?

Mots-clés : littérature, économie, consommation, plaisir, sublimation

\begin{abstract}
First examined from the point of view of economics, Literature is part of the bookindustry, the words "production" and "consumption" defining the relationship between the authors (assisted by publishers, printers, booksellers...), and the readers, whose pleasure is the final cause of the trade. But if we limit our outlook to the book in itself, it is still legitimate, considering for instance the "economy" of a narrative or of a poem, to speak of the management of the pleasure of reading, which runs from incitation to orgasm, as in a psychoanalysis of fire. It remains to ask if the sexual model may be pertinently applied to the type of gratification derived from reading. Should not we, instead, resort to the concept of sublimation to better understand reading as it is praised by Society in its valorization of socially useful activities?
\end{abstract}

Keywords: literature, economy, reading, pleasure, sublimation 
Il m'arrive de me demander pourquoi les centres de littérature et leurs diverses initiatives, au premier rang desquelles les colloques, éprouvent tant de difficultés à obtenir des financements. Une partie de la réponse figure sans doute dans le titre que les organisateurs ont donné à ces deux journées d'échanges. Nous autres littéraires avons la réputation, auprès des gens sérieux (chefs d'entreprise, banquiers, juristes, scientifiques, historiens... la liste serait interminable ), d'être des hommes et des femmes de plaisir, à peu près au même titre, mais à un degré d'éminence inférieur, que les écrivains et les artistes. Et nous courons après une légitimation de notre activité, dont la recherche de la jouissance, mi-intellectuelle, mi-esthétique, semble bien être la seule véritable raison d'être, avec, assurément, le désir de la partager.

Il est vrai que les impositions, les angoisses, les affres de la carrière universitaire, cours à préparer, travaux à diriger, concours à passer, jurys à intégrer, viennent jeter un voile de sévérité administrative sur cette justification inavouable qui se trouve à l'origine du choix que nous avons fait, à vingt ans, de devenir professeurs de littérature. Les conditions d'exercice du métier sont parfois rendues si pénibles que la source vive de notre vocation - le pur et simple plaisir de lire ( ni pur, ni simple à vrai dire ! ) - que la source s'est comme tarie.

Ainsi, hédonistes honteux et malheureux, cherchons-nous un peu de compréhension auprès de collègues d'autres facultés, qui eux se montrent libres de témoigner naïvement de leur amour de la littérature, quand ce n'est pas de nous faire part du délice sans mélange que leur a procuré tel ou tel roman classique récemment découvert ( un mathématicien me disait un jour la jubilation qu'il avait ressentie à lire en voyage La Chartreuse de Parme... ).

Nous ne trouvons de riposte à l'opprobre que dans le déni. C'est un phénomène bien significatif que la volonté qui a été la nôtre, celle de ma génération en tout cas, de rapprocher à toute force, par le formalisme, les études littéraires de la science. Qui ne se souvient de l'austérité qui pesait sur nos études à l'époque où Todorov et Genette régnaient en maîtres ? Et si Roland Barthes crut bon, avec Le Plaisir du texte, de décréter la fin du carême littéraire, c'est que lui-même avait assez mangé de ce pain dur, dont Le Degré Zéro de l'écriture conserve encore pas mal de miettes. Mais enfin, nous voici revenus, quarante ans après, à des temps meilleurs ( au moins sous cet aspect... ) : il est maintenant permis d'examiner sans gêne et sans détours la nature de l'enchantement ou, pour le moins, du contentement littéraire.

Ce n'est pas, d'ailleurs, le premier but que je vais me fixer dans les propos qui suivent. Le plaisir, littéraire ou autre, s'expérimente plus qu’il ne s'explicite. «Comme toutes les données immédiates de la conscience, le plaisir ne se définit pas; le seul moyen de le connaître est de l'éprouver » expliquait le logicien Edmond Goblot dans son Vocabulaire philosophique (1908). Que l'intelligence et la sensibilité trouvent leur compte dans la lecture d'une belle page nous suffit généralement et nous pas- 
sons outre. Certes, les neurosciences nous décrivent le détail des réseaux et des connexions neuroniques censés rendre compte de l'émotion esthétique, de ses résonances et de ses harmoniques, mais en sommes-nous véritablement plus informés sur le fin mot de l'histoire?

C'est bien plutôt du côté du livre qu'il faut regarder, comme de la culture et de la réceptivité du lecteur, sans l'opération duquel un livre reste un objet inanimé. Aussi est-il à la fois plus prudent et plus productif d'analyser le plaisir sui generis qui résulte de la lecture de tel texte singulier que d'entreprendre - comme je vais pourtant le faire - de se placer sur un plan général et abstrait. Mais pour arriver là où je souhaite poser le problème, il me faut jalonner, le plus vite possible, l'espace de pensée séparant l'immense dispositif socio-culturel qu'est l'institution littéraire de l'acte intime de lecture auquel on se livre dans une petite pièce, éventuellement sentant l'iris.

Ma démarche consistera à passer d'une vision matérialiste à une autre perspective, disons idéaliste, de la littérature, par le biais de la notion psychanalytique de sublimation. Il s'agit donc d'entrer d'abord dans une conception économique de la lecture pour en sortir bientôt, si c'est possible.

Je ne recourrai pas au marxisme pour justifier l'assimilation du livre - ou de l'œuvre d'art - à tout autre bien matériel que l'on fabrique, vend ou achète, étant entendu que la jouissance individuelle, dans une perspective capitaliste, constitue la cause finale de tout le processus. Il est curieux de noter, comme le font Wellek et Warren (1971 : 145), que Marx, quand il parle de l'art, le fait en utopiste, la valeur esthétique, qu'il voudrait universelle et à la portée de chacun, se prêtant mal à un traitement quantitatif. Non, je m’adresserai à Paul Valéry qui, dans sa «Première leçon de poétique au Collège de France » (Valéry 1957 : 1345), s'est risqué à surprendre, sinon à choquer, ses auditeurs en esquissant un parallèle entre la littérature et l'économie. Je cite :

... il me semble peut-être commode, écrit-il, d'assembler sous les seuls noms de production et de producteur, les diverses activités et les divers personnages dont nous aurons à nous occuper, si nous voulons traiter de ce qu'ils ont en commun, sans distinguer entre leurs différentes espèces. Il ne sera pas moins commode avant de spécifier que l'on parle de lecteur ou d'auditeur ou de spectateur, de confondre tous ces suppôts des œuvres de tous genre, sous le nom économique de consommateur. (Oe I: 1544)

Le mot de consommateur induit immédiatement les idées de désir et de satisfaction, et c'est tout ce dont nous avons besoin pour le moment. Comme toutes les industries liées au loisir ou au luxe, celle du livre a pour but d'éveiller et d'apaiser un appétit, plus ou moins artificiel, et de l'entretenir, plutôt que de le combler définitivement. Les éditeurs et les libraires s'entendent à merveille à gérer cette faim jamais apaisée : c'est une remarque élémentaire mais essentielle que de noter combien un 
titre accrocheur, une couverture éclatante, un papier doux au toucher et à l'odorat, une typographie aérée, disposent l'acheteur éventuel à l'acquisition d'un ouvrage. Et pourquoi, en effet, ne pas faire débuter le plaisir de lire avec ces prémices toutes sensorielles? Le reproche que l'on entend souvent faire au livre électronique a trait à son manque de matérialité (ou de palpabilité) et l'on conçoit mal qu'un bibliophile, amateur de belles reliures et de tranches colorées, se mette à collectionner des volumes virtuels, précisément parce que son plaisir est premièrement d’ordre physique. Si le livre imprimé a un avenir, c'est que le lecteur a un corps et qu'il y tient, comme au contact et à la présence dans la relation avec autrui. Gratification archaïque, assurément, mais le plaisir de lire, analogue, sans être identique, au plaisir fonctionnel de manger et de boire, durera autant que l'animal rationnel que nous sommes, c'està-dire quelques années encore...

Il me fallait au moins évoquer ces aspects mercantiles et concrets qui nous autorisent à inscrire la lecture dans le système général des échanges de richesses dont traite l'économie politique. Je n'envisagerai pas la passionnante et intrigante question des prix dans leur rapport à l'intérêt intrinsèque du livre: Don Quichotte, qui m’apportera autant, sinon bien davantage de plaisir durable que le dernier Goncourt, peut me coûter 5 euros tandis que le second en vaudra 20. C'est que la nouveauté du produit est une valeur ajoutée qui doit comme toute frivolité entrer en ligne de compte dans le calcul des plaisirs, sur lesquels spéculent les éditeurs, indépendamment des droits d'auteur.

Si l'on peut comparer l'institution littéraire à une pyramide, dont la pointe est le plaisir spirituel du Sujet lecteur, la base est formée de l'ensemble de ces facteurs et conditions matérielles qu'une représentation objective de la littérature ne peut passer sous silence. Depuis Wellek et Warren, que je mentionnais à l'instant, la bibliographie concernant l'industrialisation du livre et les méandres du circuit commercial s'est considérablement allongée: la sociologie de la littérature a cependant peu à nous apprendre du plaisir lui-même et c'est vers la psychologie qu'il convient maintenant que nous nous tournions.

Je n’abandonne pas pour autant Valéry et sa poétique. Je lui adjoins toutefois Sigmund Freud. C'est dire que je me maintiens dans les limites d'un matérialisme de bonne compagnie, en continuant de penser le plaisir littéraire en termes d'économie, non plus politique cette fois, mais psychique. On sait la prépondérance que la psychanalyse attribue au principe de plaisir dans le fonctionnement mental. Nous lisons dans le dictionnaire de Laplanche et Pontalis qu' en tant que le déplaisir est lié à l'augmentation des quantités d'excitation et le plaisir à leur réduction, le principe de plaisir est un principe économique (1967 : 332) ». Pourquoi le plaisir de lire se soustrairait-il à la mesure s’il est lui aussi identifiable à une quantité ?

Déjà Fechner soumettait les états affectifs aux algorithmes de sa psychophysique, à quoi réagissait Bergson, dans les Données immédiates de la Conscience, en 
démontrant que le plaisir et la douleur sont avant tout des qualités différentielles.

Or Paul Valéry, qui se croit si loin de Freud, semble se rallier à lui quand il expose que, dans la lecture, une énergie se dépense "à notre plus grand contentement », autrement dit que le plaisir littéraire est tout semblable au plaisir de l'acte sexuel.

Il est piquant de songer que Valéry, sans doute à l'insu de nombreux de ses auditeurs ( sauf de Jacques Lacan bien sûr... ) se soit servi de la montée du désir et de son assouvissement comme d'un modèle pour comprendre l'effet de la lecture sur le sujet qui s'y adonne. Je me permets de vous lire un autre passage de cette leçon mémorable, où le poète philosophe ne pouvant décemment se faire plus explicite ( nous sommes en 1937 au Collège de France ) se plaît, analysant l'acte de lire, à évoquer les phases de l'œuvre de chair :

L'œuvre nous offre dans chacune de ses parties, à la fois l'aliment et l'excitant. Elle éveille continuellement en nous une soif et une source. En récompense de ce que nous lui cédons de notre liberté, elle nous donne l'amour de la captivité qu'elle nous impose et le sentiment d'une sorte délicieuse de connaissance immédiate ; et tout ceci, en dépensant, à notre plus grand contentement, notre propre énergie qu'elle évoque sur un mode si conforme au rendement le plus favorable de nos ressources organiques, que la sensation de l'effort se fait elle-même enivrante, et que nous nous sentons possesseurs pour être magnifiquement possédés. Alors plus nous donnons, plus voulons-nous donner, tout en croyant de recevoir. (Valéry 1957 : 1355)

En termes d'économie du plaisir de lire, nous pourrions nous en tenir là puisque tout est dit ou suggéré : " ut orgasmos poesis »! Que la lecture littéraire soit un processus psychique - et également physique dans le cas de la lecture à haute voix consistant en la circulation et la répartition d'une énergie pulsionnelle quantifiable, susceptible d'augmentation et de diminution, Valéry, à le penser, se retrouve donc freudien sans le vouloir.

Et que nous soyons portés à le penser nous aussi, c'est ce qu'atteste tout un discours critique que nous assumons comme nôtre et qui, par réaction contre la pudibonderie du discours académique de jadis, ne craint pas d'érotiser la théorie littéraire. Kristeva, entre autres maîtres à penser, a beaucoup fait pour dévergonder grammairiens et grammairiennes. Et toute une tradition iconographique lui donne raison : je songe à ces liseuses voluptueuses représentées dans la peinture. Leur pose languissante, leur sourire rêveur, leur regard extatique ne viennent-ils pas confirmer la réalité de l'orgasme mental, cet éléphant blanc de la sexologie? Ce serait ainsi une affaire entendue et la question du plaisir de lire serait réglée par son assimilation à la jouissance sexuelle.

Nous n'en sommes pourtant pas quittes, dans la mesure où au moment même où Valéry rejoint Freud, Freud s'écarte de lui en s'avisant que l'art n'est pas entièrement 
réductible aux jeux de la pulsion sexuelle et qu'il importe, pour en rendre compte, de faire intervenir un autre processus, lui aussi économique : la sublimation. Alors que, seIon Paul Valéry, la poésie peut être comprise et expliquée par sa parenté avec le «type le plus général de l'action humaine ", en l'occurrence l'acte amoureux dans tout son développement physio-psychologique, Freud, de son côté aperçoit les limites de cette filiation directe : il y a quelque chose « au-delà du principe de plaisir ».

Je n'aborderai pas de front le si complexe problème de la sublimation chez le père de la psychanalyse, dont on sait qu'il l'a conduit, avec la découverte de l'instinct de mort, à remanier sa première représentation de l'appareil psychique. Plus encore, et pour citer à nouveau Laplanche et Pontalis : "L'absence d'une théorie cohérente de la sublimation reste une des lacunes de la pensée psychanalytique » (1967:467). Je ne suis malheureusement pas en mesure de la combler ce matin. Par contre, je déplacerai et resserrerai la question en la posant dans le cadre, moins élevé, d'une réflexion pédagogique.

Ayant été amenée au cours de ces dernières années à m’intéresser de plus près au Plan National de Lecture ( et à son extension à l'Enseignement supérieur ), j'ai eu maintes occasions de réfléchir sur les obstacles de plus en plus nombreux qui s'opposent à la fréquentation du livre, en particulier du livre littéraire, chez les jeunes. Il va sans dire que, comme enseignante, j’avais déjà été alertée par les signes récurrents d'un défaut d'appétence parmi les étudiants de littérature eux-mêmes. Mon erreur était de partir du postulat que la lecture est un plaisir qui s'offre immédiatement à tous et qu'il suffit de répéter : " plaisir, plaisir » pour que les élèves mordent à l'hameçon. Entre le constat d'échec des stratégies coutumières de motivation et le souvenir de ma pratique d'enseignante un abîme se creusait.

Je confesse avoir moi-même beaucoup joui en classe à analyser mes textes préférés et je me flatte de penser que cette mienne jouissance, n'ayant rien de simulé, a pu jouer un rôle séminal auprès de quelques-uns de mes étudiants. Qui parmi nous n'a pas ressenti cette force de propagation magnétique qui se répand comme une onde dans l'amphithéâtre? N'est-ce pas toute la magie de la parole littéraire, ou la récompense de l'effort professoral? Le charme poétique aurait donc à lui seul assez de force pour s'imposer tel quel aux esprits les plus rebelles? C'est ce dont Lucrèce semble persuadé alors qu'il cherche à entraîner le lecteur dans son élan spéculatif en quête des arides secrets de la Nature. Je le cite :

Quand les médecins veulent donner aux enfants la répugnante absinthe, ils revêtent auparavant les bords de la coupe d'une couche de miel blond et sucré; de la sorte, cet âge imprévoyant, les lèvres séduites par la douceur, avale en même temps l'amère infusion et, dupe mais non victime, en recouvre au contraire force et santé. Ainsi je fais aujourd'hui, et comme notre doctrine semble trop amère à qui ne l'a point pratiquée, comme la foule recule avec horreur devant elle, j'ai voulu te l'exposer dans l'harmonieuse 
langue des Muses et, pour ainsi dire, la parer du doux miel poétique : puissé-je ainsi tenir ton esprit sous le charme de mes vers, tandis que tu pénètres tous les secrets de la nature et les lois qui président à sa formation. (Lucrèce 1947 : 39)

Celle fonction incitatrice que Lucrèce assigne à la poésie relève d'une conception de la forme poétique comme leurre ou appât, qui sera celle du moralisme esthétique du grand Siècle. Plus généralement, c'est le doublet horacien de l'utile et de l'agréable (« docere et placere ») qui a dominé la littérature jusqu'au $19^{e}$ siècle. Les Liaisons dangereuses se veulent encore conformes à cet idéal. Emile Zola a peut-être été le dernier à vouloir concilier les deux intérêts.

Mais tandis que la littérature abandonnait la préoccupation didactique, la pédagogie a persévéré dans sa stratégie d'apprentissage par le plaisir. Je ne dirai pas qu'elle a eu tort, mais son erreur a été de penser qu'il était possible de marier harmonieusement les deux principes de plaisir et de réalité. Voici un exemple, presque cocasse, de cette illusion scolaire. Je lis, sur la couverture d'un manuel intitulé Lire à plaisir, textes et thèmes pour les classes de seconde et de première:

Ce manuel d'une conception nouvelle s'adresse aux élèves du second cycle de toutes les séries et de tous les niveaux. Tout en répondant aux nouvelles directives, il cherche à leur faire découvrir la littérature et le plaisir de lire. (Zorlu/ Yrle 1995)

Comment peut-on, sans effet comique, faire voisiner dans la même phrase les mots de la nomenclature scolaire, pour ne pas dire ministérielle ( manuel, élèves, second cycle, séries, niveaux, directives ), et celui, si nu, de plaisir : à supposer que la littérature puisse s'enseigner au collège ( les écrivains généralement en doutent), peut-elle, sans péril pour le plaisir de lire, s'entourer d'autant de précautions bureaucratiques?

Une autre tactique, plus retorse et plus efficace, consiste à faire accroire que la lecture littéraire est un fruit défendu. Je me suis longtemps servie de l'étiquette "littérature radicale " pour tenter de conduire mes étudiants vers des auteurs et des textes où les instincts les plus violents se donnent libre cours. Mettons Corneille et Lamartine sous clef pour tenter quelques voleurs. Mais comment rivaliser avec le cinéma, les séries télévisées, les jeux vidéo et autres pourvoyeurs de plaisir brut aisément accessibles? Pourquoi est-il si difficile de séduire les enfants, les adolescents - et les adultes eux-mêmes - à la lecture, si l'on prétend que l'acte de lire produit une satisfaction immédiate comme l'image?

Ne serait-il pas important de dissiper le malentendu tenant à la nature du plaisir littéraire, ce à quoi nous convie ce colloque? Quand Valéry disait qu'il n'y a pas de plaisir sans gêne, il était déjà plus proche de l'idée sur laquelle je voudrais insister pour finir. 
Je n’ai jamais défendu le maintien ou le retour du latin dans les programmes du secondaire : c'est un combat perdu depuis plus d'un siècle. Mais comment ne pas reconnaître que l'exercice de la version latine et la difficulté d'arriver au sens d'une page de Suétone pouvaient, mieux que tous nos subterfuges pédagogiques, préparer les jeunes esprits à savourer directement, sans dictionnaire ni grammaire, sans explication philologique ou historique, un chapitre de Jules Verne ou d'Alexandre Dumas? C'est aujourd'hui la mauvaise maîtrise de la langue maternelle elle-même qui empêche cette accession immédiate à la littérature, de plus en plus réservée à une élite culturelle, laquelle en vient du reste à s'interroger si elle n'est pas une survivance vouée à une disparition prochaine.

Le sujet qui nous convoque ici étant ici le plaisir de lire, je crois n'avoir plus rien à ajouter qui vaille la peine d'être écouté si, du moins, je me tiens aux termes du problème tel que je l'ai posé. Je pourrais, certes, sortir une dernière carte en introduisant l'idée de bonheur mais le bonheur n'est pas le comble du plaisir ni une suite ininterrompue de plaisirs. Qu'il y ait un bonheur de lire, une durée heureuse et tranquille de la lecture, associée à des circonstances propices, à un cadre accueillant, à une conscience pacifiée, à une confiance dans l'avenir, chacun en a fait l'expérience et garde la nostalgie de ces moments de paradis.

Mais il est trop tard pour que je m'aventure dans une psychologie du temps, sauf à renvoyer à Bachelard qui a tout dit à ce sujet: bergsonien dans l'âme (quoique non-bergsonien dans l'esprit), il sait qu'il faut commencer par être heureux pour arriver au bonheur de lire, de même que «A qui veut rêver bien, il faut dire : commencez par être heureux» (Bachelard $1960:$ II).

En revanche, je peux, en guise de péroraison, récuser l'énoncé du problème et me refuser désormais à parler de «plaisir de lire », expression entaché de dilettantisme et qui dissimule les vrais enjeux de la lecture. S’il ne s'agissait pour nous que de plaisir, le jeu en vaudra-t-il la chandelle? Car ce n'est rien moins qu'une espèce de salut que nous espérons trouver, et que nous trouvons, dans les grands livres. Le plaisir n'est pas le souverain bien, mais, au mieux, un surcroît, voire pour un janséniste un « dangereux supplément ». Franchissons donc le pas en laissant l'hédonisme pour l'eudémonisme : sans aller jusqu'à imposer la lecture comme un devoir, présentons-la, valeur socialement reconnue, pulsion sublimée, comme un acte requérant un effort et dont le plaisir qu'il peut nous apporter atteste l'accroissement de notre être et notre progrès vers sa perfection. C'est le langage d'Aristote et, dans le présent état de l'art, c'est à peu près le seul qui me paraît pouvoir être honnêtement tenu. 


\section{Note}

* Cristina Robalo-Cordeiro, née à Coimbra en 1954, est professeur titulaire de la Faculté des Lettres de l'Université de Coimbra, dont elle a été Vice-Présidente de 2003 à 20II. Présidente de l’Alliance Française de Coimbra, Vice-Présidente de l’Association Européenne d’Etudes Francophones, Secrétaire-Générale du RESUFF (réseau universitaire francophone de femmes dirigeantes dans l'enseignement supérieur), elle a exercé, de 2012 à 2016, les fonctions de Directrice du Bureau Maghreb de l'AUF, à Rabat. Elle coordonne le Plan National de Lecture pour l'Enseignement Supérieur et intègre le Comité de la Candidature de Coimbra au titre de Capitale Européenne de la Culture 2027. Auteur de Io livres (dont un roman, Fugue marocaine, et un recueil de nouvelles, Réminiscences de la lumière) de 6 traductions et de (environ) 200 articles, elle a prononcé des conférences un peu partout dans le monde (Brésil, États-Unis, Portugal, Espagne, Italie, France, Belgique, Maroc, Algérie, Tunisie, Liban, Mozambique, Côte d’Ivoire, Allemagne, Turquie, Sénégal, Burkina Faso, Luxembourg, Roumanie, Algérie, Tunisie, Canada, Abu-Dhabi, Chypre). Son œuvre critique porte en priorité sur la culture et la littérature françaises et francophones, mais elle a également publié des monographies et des articles dans le domaine de la littérature comparée et sur des auteurs de la littérature portugaise. Elle est lauréate du Prix Richelieu Senghor de la Francophonie (2008) et a été décorée de des Palmes Académiques (Officier), 200I, de la Légion d'Honneur (Chevalier), 20I4, et de l'Ordem do Infante (Commandeur), 20I5, et de l'Ordre des Arts et des Lettres (2020). Elle est Docteur Honoris Causa de l'Université Fédérale de Rio de Janeiro (UNIRIO) et a reçu le Prémio Literário Orlando Gonçalves (Menção Honrosa) pour son reccueil de nouvelles Reminiscências da Luz.

\section{Bibliographie}

Bachelard, Gaston (1960), La poétique de la rêverie, Quadrige, PUF, p. II. Goblot, Edmond (1908), Le Vocabulaire Philosophique, Paris, Armand Colin.

Laplanche, J. et Pontalis, J.-B. (1967), Vocabulaire de psychanalyse, PUF, p. 332.

Lucrèce (1947), De la Nature, Les grandes œuvres de l’Antiquité classique, Société d'édition Les Belles-Lettres, Paris, p. 39

Valéry, Paul (1957), Oeuvres I, Bibliothèque de la Pléiade, p.I345.

Wellek et Warren (1971), La théorie littéraire, Poétique/Seuil, p. 145.

Zorlu, Jacqueline et Yrle,Nicole (1995), Ellipses, 4e de couverture. 